\title{
GORRELATION BETWEEN HUMAN ABO BLOOD GROUP ANTIGENS IN SEMINAL PLASMA AND ON SEMINAL SPERMATOZOA
}

\author{
B. BOETTCHER \\ The Flinders University of South Australia, School of Biological Sciences, \\ Bedford Park, South Australia
}

(Received 4th May 1967, revised 28th September 1967)

\begin{abstract}
Summary. ABO blood group antigens on spermatozoa have been investigated using the agglutination inhibition technique. Antigens were not detected on spermatozoa from non-secretors, though they were detected on spermatozoa from secretors. Further, it has been demonstrated that spermatozoa will adsorb $\mathrm{ABO}$ antigens from aqueous solutions. The inhibition titres of seminal plasma and of spermatozoa from secretors were shown to be correlated.

It is concluded that ABO antigens are found only on seminal spermatozoa from secretors and that these are adsorbed from the seminal plasma.
\end{abstract}

\section{INTRODUCTION}

In 1964 Edwards, Ferguson and Coombs reported that they were able to get spermatozoa from secretors to participate in ABO-specific mixed agglutinations whereas those from non-secretors would not, although, in the specimens from non-secretors, non-spermatozoal cells reacted strongly. They concluded that $\mathrm{ABO}$ antigens on seminal spermatozoa are derived from the seminal plasma and that, therefore, the $\mathrm{ABO}$ antigens on spermatozoa are dependent on the secretor status of the donor. This conclusion was contrary to those of Popivanov \& Vulchanov (1962) and Shahani \& Southam (1962), who supported Gullbring's (1957) proposal that spermatozoa possess only the ABO antigens controlled by the gene they carry. It was contrary, also, to that of Levine \& Celano (1961) who reported that spermatozoa from both secretors and non-secretors possess $\mathrm{ABO}$ antigens.

Boettcher (1965) supported the conclusion of Edwards et al. (1964) since, using the agglutination inhibition technique, he had found that inhibition titres of washed sperm specimens were correlated with the titres of their respective seminal plasmas, and that the inhibition titres of sperm samples from non-secretors were not significantly different from the saline controls. Further, he showed that spermatozoa can adsorb $\mathrm{ABO}$ blood group substances from seminal plasma, since spermatozoa from an $\mathrm{O}$ non-secretor gained the capacity to inhibit a human anti-A serum and an extract of Ulex europaeus on being incubated in seminal plasma from an A secretor.

This report gives details of investigations made into the relationship between 
the amounts of $\mathrm{ABO}$ blood group antigens in seminal plasma and on seminal spermatozoa using the agglutination inhibition technique.

\section{MATERIALS AND METHODS}

Semen specimens from unknown donors were obtained from the South Australian Institute of Medical and Veterinary Science. The specimens were obtained by the Institute for testing in connection with infertility. Consequently, a number of specimens with very few spermatozoa were encountered, and discarded without examination of the antigens they possessed.

Since it was not permissible to obtain the semen donors' names and/or their blood groups, the ABO blood groups of secretor donors were determined from the seminal plasmas. The blood groups of non-secretors could not be determined.

Spermatozoa were obtained from whole semen collected in a dry jar by allowing the semen to stand for about $1 \mathrm{hr}$ to enable precipitation of many of the non-motile spermatozoa and non-spermatozoal cells, and removing the supernatant. Spermatozoa were precipitated from this by centrifugation. The precipitated spermatozoa were washed three times in physiological saline, and were then resuspended in saline. The final washing solution for each specimen was tested for its ability to inhibit an anti-human globulin serum and, if this was not negative, the specimen was re-washed.

The origins and properties of the agglutinin solutions used in this work have been described in full elsewhere (Boettcher, 1967). Human anti-A and anti-B sera with low haemolytic activity and a saline extract of Ulex europaeus seeds (anti-H) were used. No attempt was made to standardize the strengths of the anti-A and anti-B sera used, so that no comparisons of antigen concentration can be made on the basis of results using the two different antisera.

The agglutination inhibition technique, too, has been described fully elsewhere (Boettcher, 1967). Briefly, the concentration of an agglutinin solution which could be just inhibited by an equal volume of the solution under test (either seminal plasma or sperm suspension) was recorded as the inhibition titre of that solution for the particular agglutinin.

\section{RESULTS}

The agglutination inhibition titres of both seminal plasma and washed spermatozoa at a concentration of $10^{8} / \mathrm{ml}$ were determined. Because of the small quantities available, agglutination inhibition by sperm suspensions was carried out with only those agglutinins able to be inhibited by the seminal plasma, except that sperm suspensions obtained from non-secretors were tested against the lowest concentrations of all agglutinin solutions.

Preliminary studies reported elsewhere (Boettcher, 1965) were carried out on semen specimens from donors of known blood group and secretor status. However, the blood groups of the donors reported here were inferred from the ABO antigens present in the seminal plasma. Since the earlier work and the studies by Hartmann (1941) indicated that the antigens present in the seminal plasma are the same as those in the saliva, this appears to be a valid procedure. 
Consequently, the blood groups of the non-secretors involved were unable to be determined.

Of the thirty-six semen samples which were of sufficient volume and contained sufficient spermatozoa for testing, eleven did not have detectable $A B O$ blood group substances. The $\log _{2}$ of the inhibition titres of the seminal plasmas and sperm suspensions of the other twenty-five specimens, none of which was from an $\mathrm{AB}$ individual, are shown in Text-fig. 1.
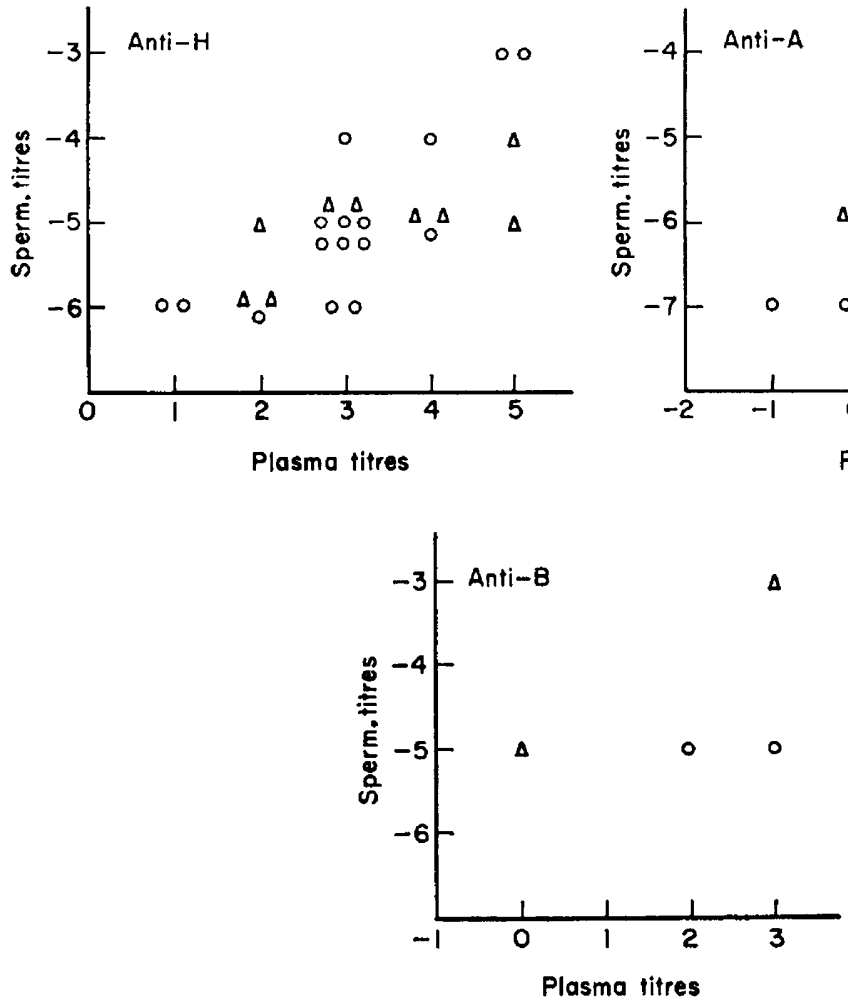

TExT-FIG. 1. $\log _{2}$ inhibition titres of seminal plasma and spermatozoa. $O$, Tests carried out on spermatozoa and seminal plasma on the day of collection; $\Delta$, tests carried out on the same day on specimens collected over a period of 3 weeks and stored deepfrozen. Eleven specimens where the seminal plasma showed no inhibition are not included.

Twenty of the thirty-six samples were tested on the days they were collected with the same set of testing solutions which were kept deep-frozen when not in use. The other sixteen specimens were deep-frozen immediately on collection over a period of 3 weeks and were thawed and tested on the one day using the same set of testing solutions as for the other semen specimens. The two sets of results are indicated differently in the figure, but there does not appear to be any difference between the results using the two techniques.

The correlation coefficient of the $\log _{2}$ inhibition titres of the seminal plasma and spermatozoa in the twenty-five semen specimens which inhibited the Ulex europaeus extract is 0.75 . This value is significantly different from 0 at the 0.001 
level $\left(t_{23}=5 \cdot 3\right)$. However, it is not significantly different from $1\left(t_{23}=1 \cdot 28\right.$. $0.30>P>0.20)$.

It is noted that the inhibition titres of $\mathrm{ABO}$ substances in seminal plasma are larger than those of $\mathrm{ABO}$ substances in saliva. A recent paper (Boettcher, 1967) reports the results of determining the $\log _{2}$ inhibition tests of salivas from group $\mathrm{O}, \mathrm{A}$ and $\mathrm{B}$ secretors using the same agglutinin solutions used here. The average $\log _{2}$ inhibition titres of the salivas and of the seminal plasmas reported here are given in Table 1 for comparison.

TABLE 1

THE AVERAGE LOG $_{2}$ INHIBITION TITRES OF SALIVAS AND SEMINAL PLASMAS FROM GROUP O, A AND B SECRETORS

\begin{tabular}{|c|c|c|c|c|c|}
\hline \multirow[b]{2}{*}{ Agglutinin solution } & \multirow[b]{2}{*}{$\begin{array}{l}\text { Blood } \\
\text { group }\end{array}$} & \multicolumn{2}{|c|}{ Salivas } & \multicolumn{2}{|c|}{ Seminal plasmas } \\
\hline & & $\begin{array}{c}\text { No. } \\
\text { tested }\end{array}$ & $\begin{array}{c}\text { Av. } \log _{2} \\
\text { inhibition } \\
\text { titre }\end{array}$ & $\begin{array}{c}\text { Noo. } \\
\text { tested }\end{array}$ & $\begin{array}{c}\text { Av. } \log _{2} \\
\text { inhibition } \\
\text { titres }\end{array}$ \\
\hline Ulex europaeus extract & $\begin{array}{l}\mathrm{O} \\
\mathrm{A} \\
\mathrm{B}\end{array}$ & $\begin{array}{r}127 \\
84 \\
35\end{array}$ & $\begin{array}{r}0.88 \pm 0.032 \\
-0.45 \pm 0.192 \\
-1.91 \pm 0.194\end{array}$ & $\begin{array}{r}11 \\
10 \\
4\end{array}$ & $\begin{array}{l}3 \cdot 73 \pm 1 \cdot 21 \\
2 \cdot 80 \pm 0 \cdot 96 \\
2 \cdot 50 \pm 1 \cdot 47\end{array}$ \\
\hline Human anti-A & A & 84 & $0.43 \pm 0.180$ & 10 & $0.60 \pm 0.37$ \\
\hline Human anti-B & B & 35 & $0.71 \pm 0.308$ & 4 & $2 \cdot 00 \pm 1 \cdot 10$ \\
\hline
\end{tabular}

The earlier report that spermatozoa from non-secretors will adsorb $\mathrm{ABO}$ antigens if they are incubated in seminal plasma from secretors (Boettcher 1965) has been confirmed a number of times in this laboratory and in others (Hay, personal communication). Further, spermatozoa have been found to take up $\mathrm{ABO}$ antigens from other aqueous fluids containing them. Spermatozoa from non-secretors, incubated in salivas from group A, B or O secretors, or solutions of purified A and B blood group substances (obtained from Commonwealth Serum Laboratories, Melbourne) and then washed thoroughly, were found to have gained the capacity to inhibit ABO agglutinin solutions.

Fluorescent-labelled antisera have been used in attempts to investigate $A B O$ antigens on spermatozoa. It is unsatisfying to report that no difference in fluorescence has been observed for spermatozoa under test and spermatozoa used as controls. Under conditions in which spermatozoa under test fluoresced, so did the controls, and where test spermatozoa would not fluoresce neither would the controls. Weil \& Rodenburg (1962) used dilute neutral formalin to eliminate non-specific fluorescence of spermatozoa during experiments with fluorescent-labelled anti-seminal plasma globulin. However, incubation of spermatozoa for $10 \mathrm{~min}$ in $10 \%$ neutral formalin followed by three washings in $0.01 \mathrm{~m}$-phosphate buffered physiological saline did not permit differentiation between test spermatozoa and controls here.

\section{DISCUSSION}

The results obtained by Edwards et al. (1964), who were able to get spermatozoa from secretors to participate in mixed agglutination but not those from 
non-secretors, and those reported here, can be interpreted as demonstrating that testicular spermatozoa do not possess $\mathrm{ABO}$ blood group antigens, but can adsorb them. Adsorption occurs with secretors when spermatozoa are ejaculated in seminal plasma possessing them, but not with non-secretors.

Before such a conclusion can be accepted it seems advisable to re-evaluate earlier reports which have provided contrary conclusions.

Gullbring (1957) claimed that, in addition to A and B antigens, his mixed cell agglutination technique detected the $\mathrm{D}$ antigen of the $\mathrm{Rh}$ series on spermatozoa. Neither Levine \& Celano (1961) nor Quinlivan \& Masouredis (1962) could confirm the presence of the $D$ antigen, and these findings have been accepted by Edwards et al. (1964) since they found it absent from leucocytes. Furthermore, the presence of the D antigen elsewhere than on erythrocytes is seriously questioned (Lawler \& Shatwell, 1962). It seems that Gullbring's use of the mixed cell agglutination technique is suspect and, since it is the technique devised by one of the authors of a paper reporting opposing results when using it (Edwards et al., 1964), it would appear unwise to accept Gullbring's conclusions.

Perhaps the lack of a satisfactory anti-B serum, which would have permitted testing of spermatozoa presumed to possess only the A antigen, led Popivanov \& Vulchanov (1962) to conclude that their sperm-agglutinating rabbit anti-A serum was able to agglutinate $A$ spermatozoa in semen from an $A B$ male, leaving B spermatozoa unagglutinated.

Edwards et al. (1964) have shown that, although spermatozoa from nonsecretors will not participate in mixed agglutination, non-spermatozoal cells in such semen specimens react strongly. The application of results obtained from antibody absorption or absorption and elution techniques on sedimented cellular deposits from semen to spermatozoa only, therefore, appears to be questionable, and these were the techniques used by Levine \& Celano (1961) when they reported finding $\mathrm{ABO}$ antigens on spermatozoa from secretors and non-secretors.

The results of the studies by Shahani \& Southam (1962), who used fluorescent antisera to examine the $A B O$ antigens on spermatozoa, should be treated with reservation. These authors concluded that there is a segregation of $A B O$ antigens on spermatozoa. However, it is expected that some of their sixteen sperm donors would have been A or B heterozygotes, since no technique designed to select homozygous individuals was mentioned and, therefore, in some cases, basing expectations on their conclusions, only half the spermatozoa in a sample would carry the $\mathrm{A}$ or the $\mathrm{B}$ antigen. Thus, when using fluorescent-labelled anti-A or anti-B only half the spermatozoa would be expected to fluoresce. No mention is made of this. Further, the coloured plates are sufficient in themselves to show this point. In the plate showing spermatozoa from an $A B$ male treated with FITc-labelled anti-A (green) and RITc-labelled anti-B (orange), the numbers of spermatozoa of the two colours are (approx.) twenty and fifty. With a hypothesis of equal numbers of spermatozoa of the two colours the value for $\chi^{2}$ is $<12.9, P<0.001$. The two plates showing spermatozoa from an A male treated with FrTc-labelled anti-A and spermatozoa from a B male treated with RITClabelled anti-B, show all spermatozoa fluorescing with equal intensity. Under 
the conclusion of the authors, this would indicate that both semen specimens came from homozygous donors, the probability of which, assuming random selection of sperm donors, is less than $1 \%$.

It seems reasonable, therefore, now to conclude that $\mathrm{ABO}$ antigens are to be found only on seminal spermatozoa from secretors and that these are adsorbed from the seminal plasma.

\section{ACKNOWLEDGMENTS}

Much of this work has been recorded in the Ph.D. thesis Genetical control of blood group substances in body secretions, Adelaide, 1965, undertaken whilst the author held a Reserve Bank of Australia Fiftieth Anniversary Postgraduate Scholarship. The advice and guidance of Dr M. J. Mayo and the support and encouragement of Professor J. H. Bennett are very gratefully acknowledged.

\section{REFERENCES}

BOETTCHER, B. (1965) Human ABO blood group antigens on spermatozoa from secretors and nonsecretors. 7. Reprod. Fert. 9, 267.

Bötтcher, B. (1967) Correlations between inhibition titres of blood group substances in salivas from $\mathrm{A}_{1}, \mathrm{~A}_{2}$ and B secretors. Aust. F. exp. Biol. med. Sci. 45, 495.

Edwards, R. G., Ferguson, L. C. \& Coomrs, R. R. A. (1964) Blood group antigens on human spermatozoa. 7. Reprod. Fert. 7, 153.

GullbRing, B. (1957) Investigation on the occurrence of blood group antigens in spermatozoa from man, and serological demonstration of the segregation of characters. Acta med. scand. 159, 169.

Hartmann, G. (1941) Group antigens in human organs. Munksgaard, Copenhagen.

Lawler, S. D. \& Shatwell, H. S. (1962) Are Rh antigens restricted to red cells? Vox Sang. 7, 488.

Levine, P. \& Celano, M. J. (1961) The question of $\mathrm{D}\left(\mathrm{Rh}_{\mathrm{o}}\right)$ antigenic sites on human spermatozoa. Vox Sang. 6, 720.

Popivanov, R. \& Vulchanov, V. H. (1962) Segregation of man's AB-group spermatozoa in A- and B-spermatozoa through agglutination with immune anti-A rabbit serum. Z. ImmunForsch. 124, 206.

Quinlivan, W. L. G. \& Masouredis, S. P. (1962) $\mathrm{Rh}_{0}(\mathrm{D})$ antigen content of human spermatozoa. Immunology, 5, 267.

Shahani, S. \& Southam, A. L. (1962) Immunofluorescent study of the ABO blood group antigens in human spermatozoa. Am. F. Obstet. Gynec. 84, 660.

WeIL, A. J. \& Rodenburg, J. M. (1962) The seminal vesicle as the source of the spermatozoa-coating antigen of seminal plasma. Proc. Soc. exp. Biol. Med. 109, 567. 